\title{
A CRISPR-based base-editing screen for the functional assessment of BRCA1 variants
}

\author{
Jiyeon Kweon ${ }^{1,2} \cdot$ An-Hee Jang ${ }^{1,2} \cdot$ Ha Rim Shin ${ }^{1,2} \cdot$ Ji-Eun See $^{1,2} \cdot$ Woochang Lee ${ }^{3} \cdot$ Jong Won Lee ${ }^{4}$ \\ Suhwan Chang ${ }^{1} \cdot$ Kyunggon $\mathrm{Kim}^{5,6} \cdot$ Yongsub Kim $\mathbb{1}^{1,2}$
}

Received: 17 January 2019 / Revised: 31 May 2019 / Accepted: 15 June 2019 / Published online: 29 August 2019

(c) The Author(s) 2019. This article is published with open access

\begin{abstract}
Genetic mutations in BRCAl, which is crucial for the process of DNA repair and maintenance of genomic integrity, are known to increase markedly the risk of breast and ovarian cancers. Clinical genetic testing has been used to identify new $B R C A 1$ variants; however, functional assessment and determination of their pathogenicity still poses challenges for clinical management. Here, we describe that CRISPR-mediated cytosine base editor, known as BE3, can be used for the functional analysis of BRCAl variants. We performed CRISPR-mediated base-editing screening using 745 gRNAs targeting all exons in BRCAl to identify loss-of-function variants and identified variants whose function has heretofore remained unknown, such as c. $-97 \mathrm{C}>\mathrm{T}$, c. $154 \mathrm{C}>\mathrm{T}$, c. $3847 \mathrm{C}>\mathrm{T}$, c.5056C $>\mathrm{T}$, and c.4986+5G $>\mathrm{A}$. Our results show that CRISPR-mediated base editor is a powerful tool for the reclassification of variants of uncertain significance (VUSs) in BRCAl.
\end{abstract}

\section{Introduction}

The breast cancer type 1 susceptibility gene (BRCAl) is a tumor suppressor gene related to the maintenance of genome integrity [1]. Inherited loss-of-function (LOF) mutations of

Supplementary information The online version of this article (https:// doi.org/10.1038/s41388-019-0968-2) contains supplementary material, which is available to authorized users.

Yongsub Kim

yongsub1.kim@gmail.com

1 Department of Biomedical Sciences, Asan Medical Institute of Convergence Science and Technology, Asan Medical Center, University of Ulsan College of Medicine, Seoul, Republic of Korea

2 Stem Cell Immunomodulation Research Center, University of Ulsan College of Medicine, Seoul, Republic of Korea

3 Department of Laboratory Medicine, Asan Medical Center, University of Ulsan College of Medicine, Seoul, Republic of Korea

4 Division of Breast Surgery, Department of Surgery, Asan Medical Center, University of Ulsan College of Medicine, Seoul, Republic of Korea

5 Department of Convergence Medicine, Asan Medical Center, University of Ulsan College of Medicine, Seoul, Republic of Korea

6 Clinical Proteomics Core Lab, Asan Institute for Life Sciences, Seoul, Republic of Korea
BRCAl confer susceptibility to breast, ovarian, prostate, and pancreatic cancer; therefore, the identification and functional assessment of BRCAl variants is important for the clinical management of various diseases [2]. Advances in sequencing technology led to the identification of many BRCAl variants through clinical genetic testing. To investigate the pathogenicity of these BRCAl variants, various functional assessment methods have been developed, including fluorescent reporter assays, embryonic stem cell viability assays, and therapeutic drug-based sensitivity assays [3]. These assays utilize exogenously expressed $B R C A l$ variants and have clarified the function of a lot of BRCAl variants, however, the exogenous expression often result in their overexpression, which can affects gene dosage, protein folding, complex assembly, and downstream regulation. Furthermore, these assays cannot be applied to the posttranscriptional regulation such as mRNA splicing, transcript stability, and effect of untranslated region [4].

Engineered nucleases, represented by the CRISPR-Cas9 system, were developed for targeted genome editing in living cells and organisms [5]. The CRISPR-Cas9 system induces chromosomal DNA double-strand breaks in a target sequence-specific manner, which are repaired via errorprone nonhomologous end joining or error-free homologydirected repair (HDR), resulting in gene disruption, addition, and correction. CRISPR-mediated HDR can be used to introduce point mutations; however, it typically induces 
a

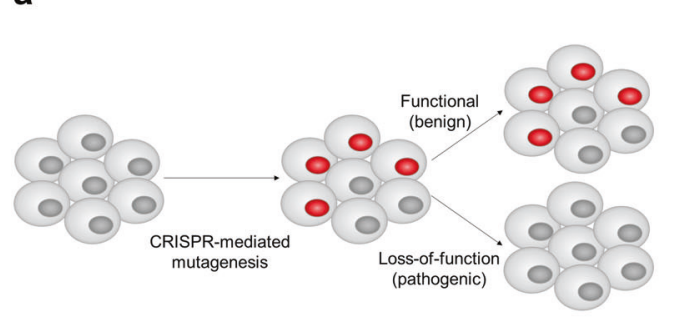

b

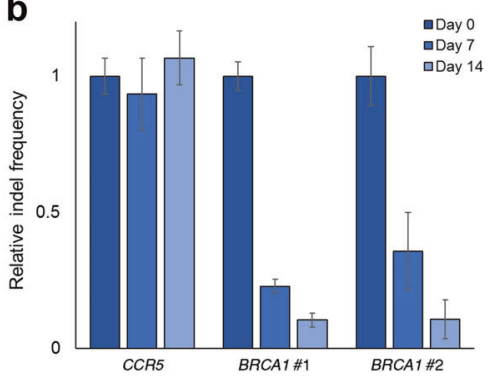

C

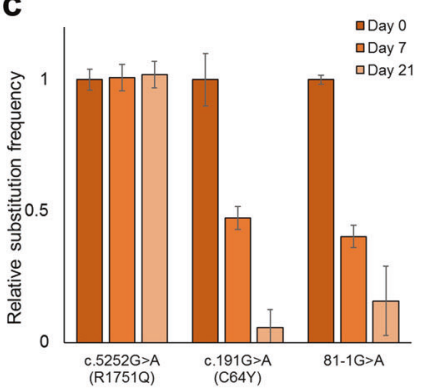

f

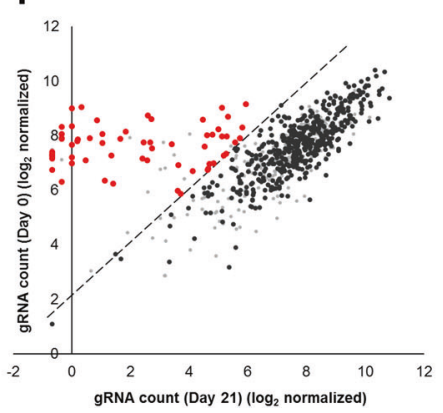

Fig. 1 Functional assessment of $B R C A 1$ variants using CRISPR-based base editing. a Schematic overview of the functional analysis of $B R C A 1$ via targeted mutagenesis. b Cell viability analysis of HAP1Cas9 cells transfected with two different gRNAs targeting BRCA1 using targeted deep sequencing. BRCA1 \#1 and BRCA1 \#2 indicate each $B R C A 1$-targeting gRNA, and the $C C R 5$-targeting gRNA was used as a negative control. $\mathbf{c}$ Cell viability analysis of HAP1-BE3 cells transfected with gRNAs targeting pathogenic mutations [c.81-1G>A and c.191G $>$ A (p.C64Y)] and a benign mutation [c.5252G $>A$ (p.R1751Q)] using targeted deep sequencing. d Timeline of BRCAI variant screens in HAP1-Cas9 and -BE3 cells. e Box plot showing the distribution of gRNA frequencies at different time points after gRNA transduction. f Scatterplot showing the depletion of specific gRNAs after 21 days. Error bars show the standard error of the mean unwanted insertion and deletion (indel) mutations. In addition, because the system shows low efficiency and requires homologous DNA templates, it is inadequate for introducing various mutations into large genes such as BRCA1 or BRCA2.

Recently, several groups have shown that Cas9 nickase or catalytically inactive Cas9 (dead Cas9, dCas9) fused with cytidine deaminase induces target-specific nucleotide substitutions in live cells and organisms in the absence of homologous DNA templates [6-9].

Here, we used the Base Editor 3 (BE3), which induces targeted $\mathrm{C}: \mathrm{G}$ to $\mathrm{T}: \mathrm{A}$ conversions, for the functional assessment of BRCA1 variants, and identified the pathogenicity of BRCAl variants with unknown functions through BE3-mediated high-throughput screens.

\section{Results}

Because $B R C A 1$ plays an important role in the process of HDR, LOF of BRCAl affects cell viability, and this property can be used to evaluate the function of $B R C A 1$. In other words, the introduction of $B R C A l$ variants with LOF mutations into a cell results in cell death with increasing passage numbers, and this can be detected through analysis of mutation frequencies (Fig. 1a).

The HAP1 cell is widely used in genetic screens, because it is nearly-haploid cell lines, and LOF can be effectively introduced into the cells $[10,11]$. We, first, examined whether functional assessment of BRCAl is possible in the HAP1 cell line. We generated Cas9-expressing HAP1 (HAP1-Cas9) and BE3-expressing HAP1 (HAP1-BE3) cell lines by infecting HAP1 cell lines with lentiviral particles expressing Cas9 or BE3. The genome editing activities of single clones were then analyzed to select highly active clones. The clones HAP1-Cas9 \#7 and HAP1-BE3 \#5 were chosen for further study (Supplementary Fig. 1).

As a proof-of-concept, BRCA1-targeting gRNAs were transfected into HAP1-Cas9 cell lines to disrupt BRCA1 and the cells were cultured for 14 days with $0.5 \mu \mathrm{M}$ olaparib, inhibitor of poly ADP ribose polymerase (PARP), which increases the sensitivity of BRCA1 deficiency [12]. The mutation frequencies were measured by targeted deep sequencing, and the relative indel frequencies were significantly decreased with time (Fig. 1b).

HAP1-BE3 cell lines were used to induce pathogenic mutations disrupting BRCAl function by nucleotide substitutions. Three different mutations (c.81-1G>A, c. $191 \mathrm{G}>\mathrm{A}$, and c.5252G $>\mathrm{A}$ ) were introduced into HAP1- 

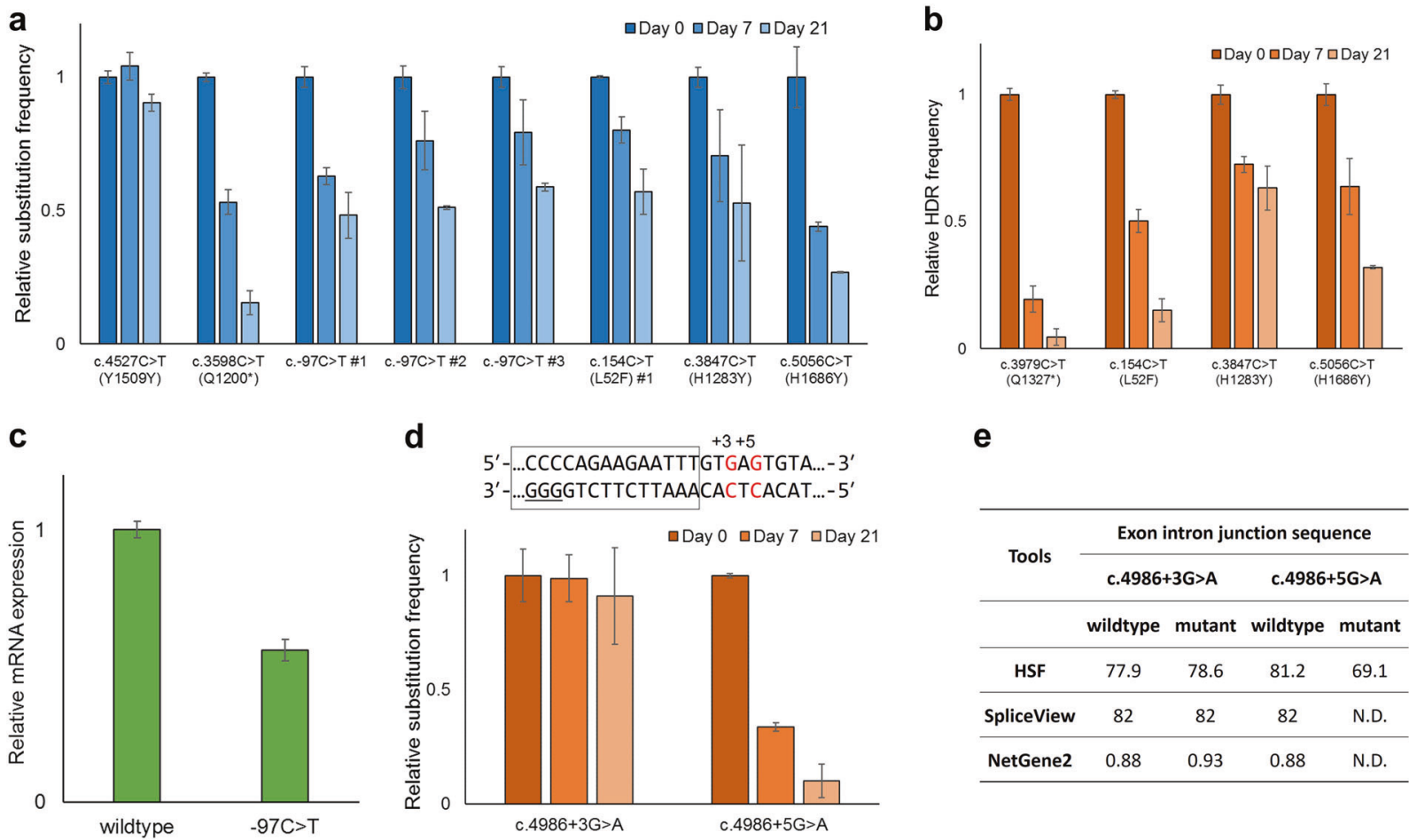

d

$5^{\prime}-. .$. CCCCAGAAGAATTTGTGAGTGTA...- $3^{\prime}$ $3^{\prime}$-...GGGGTCTTCTTAAACACTCACAT...- $5^{\prime}$

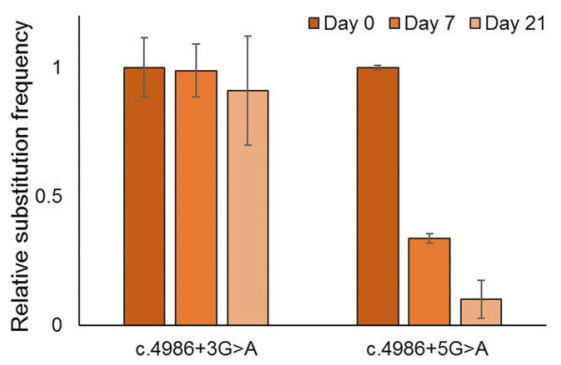

e

\begin{tabular}{ccccc}
\hline \multirow{2}{*}{ Tools } & \multicolumn{3}{c}{ Exon intron junction sequence } \\
\cline { 2 - 5 } & \multicolumn{2}{c}{ c.4986+3G $>\mathrm{A}$} & \multicolumn{2}{c}{ c.4986+5G $>\mathrm{A}$} \\
\hline & wildtype & mutant & wildtype & mutant \\
\hline HSF & 77.9 & 78.6 & 81.2 & 69.1 \\
\hline SpliceView & 82 & 82 & 82 & N.D. \\
\hline NetGene2 & 0.88 & 0.93 & 0.88 & N.D. \\
\hline
\end{tabular}

Fig. 2 Validation of individual $B R C A 1$ variants inducing $B R C A l$ dysfunction. a Cell viability analysis of HAP1-BE3 cells transfected with each candidate gRNA using targeted deep sequencing. The gRNAs inducing c.4527C $>\mathrm{T}$ and c. $3598 \mathrm{C}>\mathrm{T}$ were used as a negative and a positive control, respectively. b Functional validation of BRCA1 variants using the CRISPR-based HDR method. c 5'-UTR reporter assays confirming the transcriptional repression of BRCAl by the
$-97 \mathrm{C}>\mathrm{T}$ mutation. $\mathbf{d}$ Cell viability analysis of the intronic mutations c. $4986+3 \mathrm{G}>\mathrm{A}$ and c. $4986+5 \mathrm{G}>\mathrm{A}$ induced by a single gRNA. Targetable $C: G$ pairs and PAM are in red and underlined, respectively. Exon is shown as a rectangle. e In silico analysis of c.4986+3G $>$ A and c. $4986+5 \mathrm{G}>$ A using Human Splicing Finder, SpliceView, and NetGene2. Duplicate wells for each gRNA at each time point were processed. Error bars show the standard error of the mean
BE3 cells, and the cells were cultured for 14 days with $0.5 \mu \mathrm{M}$ olaparib [13] (Fig. 1c). The relative substitution frequencies of c.81-1G $>\mathrm{A}$ and c.191G $>\mathrm{A}$ (p.C64Y) BRCA1 mutations, known pathogenic variants, were decreased, whereas that of the likely benign missense variant, c.5252G >A (p.R1751Q), was retained. These results indicated that functional assessment of BRCAl can be performed in HAP1 cell lines, as reported previously [14], and HAP1-BE3 cell lines can be useful for introducing desired mutations into endogenous BRCA1.

Next, we designed a pooled gRNA library targeting BRCA1 to perform CRISPR-based high-throughput screens. We selected 745 gRNAs that could target all exon sequences and $15 \mathrm{bp}$ sequences around the exon-intron junction. Among the 745 gRNAs, 533 gRNAs can induce $\mathrm{C}: \mathrm{G}$ to $\mathrm{T}: \mathrm{A}$ conversions and a total of $660 \mathrm{C}: \mathrm{G}$ to $\mathrm{T}: \mathrm{A}$ conversions can be generated by 533 gRNAs in BRCAl. These gRNAs were synthesized as pooled oligonucleotides and subcloned into lentiviral vectors via isothermal assembly. To determine whether the relative abundance of gRNAs could be used as a readout for BRCA1 functional assessments, as in most CRISPR-based high-throughput screens [15], we transduced gRNAs capable of introducing pathogenic mutations into $B R C A 1$, and both the relative abundance of gRNAs and endogenous mutations were analyzed. For the high-throughput functional assessment of $B R C A 1$, the pooled gRNA library was transduced into HAP1-Cas9 or HAP1-BE3 cells with an MOI of 0.3 , and the infected cells were cultured for 21 days with $0.5 \mu \mathrm{M}$ olaparib, a PARP inhibitor with synthetic lethality in BRCA1-deficient cells (Fig. 1d). The change in the relative abundance of gRNAs was analyzed by targeted deep sequencing of three biological triplicates of genomic DNA.

The relative abundance of $24.2 \%$ of gRNAs (180 of the 745 gRNAs) in HAP1-Cas9 cells and $8.1 \%$ of gRNAs (60 of the 745 gRNAs) in HAP1-BE3 cells decreased after 21 days (fold change $>4 ; p<0.05$; FDR $<0.25$ ) (Fig. 1e, f and Supplementary Fig. 2). Unlike HAP1-Cas9 cells, which disrupt BRCAl by frameshift mutations, HAP1-BE3 cells induced LOF of BRCAl through nucleotide substitution, and relatively few gRNAs were depleted. We analyzed the potential off-target effect of each of 60 gRNAs using CasOFFinder and excluded gRNAs targeting more than three loci, resulting in the identification of $27 \mathrm{gRNAs}$ as candidates for the induction of BRCAl dysfunction (Supplementary Table 1) [16]. The target positions of the 27 
gRNAs were not limited to specific exons but were distributed among various exons of BRCAl (Supplementary Fig. 3). The BRCAl variants that could be induced by each of the 27 gRNAs were identified in the ClinVar database, which showed that 13 gRNAs introduced known pathogenic mutations in BRCAl [13].

Based on the results of high-throughput screens, we selected six gRNAs for further functional assessment of each BRCA1 variant; three gRNAs for c. $-97 \mathrm{C}>\mathrm{T}$, one gRNA each for c. $154 \mathrm{C}>\mathrm{T}$, c. $3847 \mathrm{C}>\mathrm{T}$, and c.5056C $>\mathrm{T}$. The c.3598C $>\mathrm{T}$ and c.4527C $>\mathrm{T}$ inducing gRNAs were used as positive and negative controls, respectively. Each gRNA was cloned into a plasmid DNA vector and transfected into HAP1-BE3 cells, and the endogenous BRCAI mutations induced by the gRNAs were tracked by targeted deep sequencing for 21 days (Fig. 2a and Supplementary Fig. 4). The relative mutation frequencies of c.3598C $>$ T (p. Q1200*), a pathogenic variant, dramatically decreased, whereas those of c.4527C>T (p.Y1509Y), the benign variant, remained similar. We analyzed three $B R C A 1$ variants, c. $154 \mathrm{C}>\mathrm{T}$ (L52F) in the RING domain, c.3847C $>\mathrm{T}$ $(\mathrm{H} 1283 \mathrm{Y})$ in the SQ/TQ cluster domain, and c.5056C $>\mathrm{T}$ (H1686Y) in the BRCT domain, which are reported as variants of uncertain significance (VUSs) in the ClinVar database. The relative mutation frequencies of the three variants were decreased in a time-dependent manner, and the function of c.154C $>$ T (L52F), c.3847C $>$ T (H1283Y), and c.5056C $>\mathrm{T}(\mathrm{H} 1686 \mathrm{Y})$ was verified by CRISPRmediated HDR (Fig. 2b). The results suggested that these three VUSs affected BRCAI function and could be categorized as pathogenic mutations. Analysis of the c. $-97 \mathrm{C}>\mathrm{T}$ variant in the $5^{\prime}$-UTR region, which could be induced by three different gRNAs, showed that the relative mutation frequencies were significantly reduced in cells transfected with the three gRNAs. The 5'-UTR region of BRCAl might regulate the transcription level, and several mutations in the $5^{\prime}$-UTR region are known as pathogenic variants. To further validate the c. $-97 \mathrm{C}>\mathrm{T}$ variant, we performed a luciferase reporter assay in HEK293T/17 cells, which showed that the c. $-97 \mathrm{C}>\mathrm{T}$ mutation in the $5^{\prime}$-UTR caused a twofold downregulation of gene expression (Fig. 2c). This led to the identification of a novel potentially pathogenic mutation in the 5'-UTR region of $B R C A l$ and suggested the importance of the UTR region for clinical genetic testing. We additionally confirmed the c. $4986+3 \mathrm{G}>\mathrm{A}$ and $c .4986+5 \mathrm{G}>\mathrm{A}$ variants induced by a gRNA targeting the splicing junction. As shown in Fig. 2d, the relative mutation frequencies of the c. $4986+5 \mathrm{G}>\mathrm{A}$ variant decreased with time, whereas those of the c. $4986+3 \mathrm{G}>\mathrm{A}$ variant remained similar. In silico analysis showed that only c. $4986+5 \mathrm{G}>\mathrm{A}$, but not c. $4986+3 \mathrm{G}>\mathrm{A}$, disrupted the splicing donor site; therefore, the $c .4986+5 \mathrm{G}>\mathrm{A}$ variant, which was previously reported as a VUS, could be classified as a pathogenic variant (Fig. 2e) [17, 18].

\section{Discussion}

In summary, this paper describes the first application of the BE3 system for the functional assessment of BRCA1 and the successful development of a high-throughput CRISPRmediated base-editing screen for the identification of LOF variants in BRCA1. CRISPR-mediated base-editing screens identified several VUSs, including c.154C $>\mathrm{T}$ (L52F), c.3847C $>\mathrm{T}$ (H1283Y), c.5056C $>\mathrm{T}$ (H1686Y), and c.4986 $+5 \mathrm{G}>\mathrm{A}$, that disrupt the function of BRCAl, and these variants might be classified as pathogenic mutations.

Komor et al. developed BE3 and demonstrated that it could be used to introduce several disease-relevant mutations into mammalian cells [6]. We performed highthroughput functional assessment of BRCAl using the BE3 system and determined the lethality of dysfunctions in $B R C A 1$, which is an approach that eliminates the need to create individual mutant cell lines. Findlay et al. recently developed a saturation genome editing (SGE) method based on CRISPR-mediated HDR and used it for the functional assessment of 4000 single nucleotide variants (SNVs) in 13 small exons of BRCAI [14, 19]. Compared to our baseediting screens, the SGE method has no limitation regarding the target positions of SNVs because of the use of an artificial homologous donor DNA template. However, the SGE method is based on CRISPR-mediated HDR, and it is therefore difficult to use for screening large genes because of limitations in the production of donor DNA template. Although the base editor is limited regarding the gRNA design and C:G-to-T:A conversion, the development of the CRISPR-Cas system (e.g., CRISPR-based adenosine base editing [20], Cas variants with altered PAM sequences $[5,21,22])$ extends the use of base-editing screens for biomedical research.

\section{Methods}

\section{Construction of plasmid DNA}

spCas9 in lentiCas9-Blast (Addgene plasmid \#52962) and BE3 in pCMV-BE3 (Addgene plasmid \#73021) were used for genome editing. To construct the lentiBE3-Blast plasmid DNA for lentivirus production, the BE3 coding regions were amplified by PCR and cloned into the lentiCas9-Blast vector using XbaI (NEB \#R0145) and BamHI (NEB \#R3136) restriction enzymes. The gRNA constructs were cloned into lentiGuide-puro (Addgene plasmid \#52963), 
and the target sequences of each gRNA are listed in Supplementary Table 2 .

\section{Design and construction of gRNA library}

To design BRCA1-targeting gRNAs, exon sequences and the $15 \mathrm{bp}$ regions around the sequences of exon-intron junctions were obtained from GenBank at NCBI [23]. Then, all possible target sites with $5^{\prime}-(\mathrm{N})_{\times 20}-\mathrm{NGG}-3^{\prime}$ and $5^{\prime}-\mathrm{CCN}-(\mathrm{N})_{\times 20^{-}}$ $3^{\prime}$ were searched using Cas-Designer (http://www.rgenome. net/cas-designer/) and 745 gRNAs-targeting BRCAl were listed. To generate a gRNA library, pooled oligonucleotides containing the coding sequences of the gRNAs were synthesized (Custom Array Inc.) and cloned into the lentiGuidepuro vector as previously described [24].

\section{Cell culture and construction of Cas9- or BE3- expressing cell lines}

HAP1 cells were maintained in Iscove's modified Dulbecco's medium with $10 \%$ fetal bovine serum (FBS) and $1 \%$ penicillin/streptomycin at $37^{\circ} \mathrm{C}$ and $5 \% \quad \mathrm{CO}_{2}$, and HEK293T/17 cells were maintained in Dulbecco's modified Eagle's medium with $10 \%$ FBS and $1 \%$ penicillin/streptomycin at $37{ }^{\circ} \mathrm{C}$ and $5 \% \mathrm{CO}_{2}$. To generate HAP1-Cas 9 and HAP1-BE3 cells, lentiviral particles were generated with $15 \mu \mathrm{g}$ of lentiCas9-Blast (or lentiBE3-Blast) and two viral packaging plasmids ( $9 \mu \mathrm{g}$ of psPAX2 and $6 \mu \mathrm{g}$ of pMD2.G) as previously described and transduced into HAP1 cells with an MOI of 0.1 [25]. The infected HAP1 cells were selected on media containing $10 \mu \mathrm{g} / \mathrm{mL}$ blasticidin, and single clones were isolated. To select HAP1-Cas9 and HAP1-BE3 single clones, each of the seven clones of HAP1-Cas9 and HAP1-BE3 were infected with CCR5 targeting gRNAs, and mutation frequencies were analyzed by the T7 endonuclease I (T7E1) assay and targeted deep sequencing as previously described [26]. Among them, the highly active single clones (HAP1-Cas9 \#7 and HAP1-BE3 \#5) were selected and used in this study (Supplementary Fig. 1)

\section{Base-editing screen and analysis}

For gRNA library screening, $2 \times 10^{6}$ HAP1-Cas9 or HAP1BE3 cells were seeded into six-well plates, and lentiviral particles of the gRNA library were infected with an MOI of 0.3 . The infected cells were selected on medium containing $1 \mu \mathrm{g} / \mathrm{mL}$ puromycin. After 7 days of puromycin selection, at least $5 \times 10^{5}$ cells were collected to measure the frequency of each gRNA in the initial pool (Day 0 ), and $1 \times 10^{6}$ cells were maintained with $0.5 \mu \mathrm{M}$ olaparib. After 7 and 21 days, the cells were collected, and genomic DNA was isolated using the DNeasy Blood \& Tissue Kit (Qiagen, Cat. No: 69504) for analysis by targeted deep sequencing as previously described. The screening data were analyzed using Count_space.py and the pipeline MAGeCK (ver. 0.5.6). In BE3-based screens, 212 gRNAs with no $\mathrm{C}$ in the targetable range of each gRNA were used as non-target controls. Positions of coding nucleotides and amino acids in BRCAI are referenced by ClinVar transcript annotation for BRCA1, transcript NM_007294.3 (NCBI).

\section{Targeted deep sequencing analysis}

Twenty-four hours before transfection, $5 \times 10^{5} \mathrm{HAP} 1$ (-Cas9 or -BE3) cells were seeded in 24-well plates or $1 \times 10^{5}$ HAP1(-Cas9 or -BE3) cells were seeded in 96-well plates (Corning). All transfection experiments were conducted using FuGENE HD (Promega, Cat. No: E2311) according to the manufacturer's protocol. Three days after transfection, genomic DNAs were isolated from each well using the DNeasy Blood \& Tissue Kit (Qiagen) and used as the Day 0 sample. To analyze endogenous mutation frequencies, the target regions in genomic DNAs were amplified with appropriate primers using Phusion DNA polymerase (New England Biolabs) according to the manufacturer's protocol. The PCR amplicons were confirmed using $2 \%$ agarose gel electrophoresis and subjected to Illumina MiniSeq. The sequencing data were analyzed using Cas-Analyzer (http://www.rgenome.net/cas-analyzer/). The number of sequence reads are shown in Supplementary Table 3. The PCR primer sequences are listed in Supplementary Table 4.

\section{Luciferase assay}

To construct the BRCA1 5'-UTR reporters, the $232 \mathrm{bp}$ wildtype and variant sequences were cloned into pGL4.20 with the CMV promoter. The luciferase reporters were transfected into HEK293T/17 cells, and luciferase activities were measured with the Dual-Luciferase ${ }^{\circledR}$ Reporter Assay System (Promega Cat. No: E1960) according to the manufacturer's protocol.

\section{Data availability}

The deep sequencing data are available at the NCBI Sequence Read Archive (SRA) under accession number PRJNA529534.

Acknowledgements We thank Jisun Kim and Sae Byul Lee for helpful discussions and insightful comments on the paper. We thank members at Center for Genome Engineering, Institute for Basic Science for assistance and resources for high-throughput sequencing. We thank the Scientific Publications Team at Asan Medical Center for editorial assistance in preparing the paper. 
Funding This work was supported by the National Research Foundation of Korea (grants 2017M3A9B4062419, 2019R1F1A1057637, and 2018R1A5A2020732 to Y.K.), the Korean Health Technology R\&D Project, Ministry of Health and Welfare, Republic of Korea (grant HI17C0337 to Y.K.), and Asan institute for Life Sciences, Republic of Korea (grants 2018-784, and 2018-725 to Y.K.).

\section{Compliance with ethical standards}

Conflict of interest The authors declare that they have no conflict of interest.

Publisher's note: Springer Nature remains neutral with regard to jurisdictional claims in published maps and institutional affiliations.

Open Access This article is licensed under a Creative Commons Attribution 4.0 International License, which permits use, sharing, adaptation, distribution and reproduction in any medium or format, as long as you give appropriate credit to the original author(s) and the source, provide a link to the Creative Commons license, and indicate if changes were made. The images or other third party material in this article are included in the article's Creative Commons license, unless indicated otherwise in a credit line to the material. If material is not included in the article's Creative Commons license and your intended use is not permitted by statutory regulation or exceeds the permitted use, you will need to obtain permission directly from the copyright holder. To view a copy of this license, visit http://creativecommons. org/licenses/by/4.0/.

\section{References}

1. Roy R, Chun J, Powell SN. BRCA1 and BRCA2: different roles in a common pathway of genome protection. Nat Rev Cancer. 2011;12:68-78.

2. Kuchenbaecker KB, Hopper JL, Barnes DR, Phillips KA, Mooij TM, Roos-Blom MJ, et al. Risks of breast, ovarian, and contralateral breast cancer for BRCA1 and BRCA2 mutation carriers. JAMA. 2017;317:2402-16.

3. Millot GA, Carvalho MA, Caputo SM, Vreeswijk MP, Brown MA, Webb M, et al. A guide for functional analysis of BRCA1 variants of uncertain significance. Hum Mutat. 2012;33:1526-37.

4. Gibson TJ, Seiler M, Veitia RA. The transience of transient overexpression. Nat Methods. 2013;10:715-21.

5. Knott GJ, Doudna JA. CRISPR-Cas guides the future of genetic engineering. Science. 2018;361:866-9.

6. Komor AC, Kim YB, Packer MS, Zuris JA, Liu DR. Programmable editing of a target base in genomic DNA without doublestranded DNA cleavage. Nature. 2016;533:420-4.

7. Kim K, Ryu SM, Kim ST, Baek G, Kim D, Lim K, et al. Highly efficient RNA-guided base editing in mouse embryos. Nat Biotechnol. 2017;35:435-7.
8. Park DS, Yoon M, Kweon J, Jang AH, Kim Y, Choi SC. Targeted base editing via RNA-guided cytidine deaminases in Xenopus laevis embryos. Mol Cells. 2017;40:823-7.

9. Hess GT, Fresard L, Han K, Lee CH, Li A, Cimprich KA, et al. Directed evolution using dCas9-targeted somatic hypermutation in mammalian cells. Nat Methods. 2016;13:1036-42.

10. Blomen VA, Majek P, Jae LT, Bigenzahn JW, Nieuwenhuis J, Staring J, et al. Gene essentiality and synthetic lethality in haploid human cells. Science. 2015;350:1092-6.

11. Wang T, Birsoy K, Hughes NW, Krupczak KM, Post Y, Wei JJ, et al. Identification and characterization of essential genes in the human genome. Science. 2015;350:1096-101.

12. Farmer H, McCabe N, Lord CJ, Tutt AN, Johnson DA, Richardson TB, et al. Targeting the DNA repair defect in BRCA mutant cells as a therapeutic strategy. Nature. 2005;434:917-21.

13. Landrum MJ, Lee JM, Benson M, Brown G, Chao C, Chitipiralla $\mathrm{S}$, et al. ClinVar: public archive of interpretations of clinically relevant variants. Nucleic Acids Res. 2016;44:D862-868.

14. Findlay GM, Daza RM, Martin B, Zhang MD, Leith AP, Gasperini $\mathrm{M}$, et al. Accurate classification of BRCA1 variants with saturation genome editing. Nature. 2018;562:217-22.

15. Kweon J, Kim Y. High-throughput genetic screens using CRISPR-Cas9 system. Arch Pharm Res. 2018;41:875-84.

16. Bae S, Park J, Kim JS. Cas-OFFinder: a fast and versatile algorithm that searches for potential off-target sites of Cas9 RNAguided endonucleases. Bioinformatics. 2014;30:1473-5.

17. Brunak S, Engelbrecht J, Knudsen S. Prediction of human mRNA donor and acceptor sites from the DNA sequence. J Mol Biol. 1991;220:49-65.

18. Desmet FO, Hamroun D, Lalande M, Collod-Beroud G, Claustres M, Beroud C. Human Splicing Finder: an online bioinformatics tool to predict splicing signals. Nucleic Acids Res. 2009;37:e67.

19. Findlay GM, Boyle EA, Hause RJ, Klein JC, Shendure J. Saturation editing of genomic regions by multiplex homologydirected repair. Nature. 2014;513:120-3.

20. Gaudelli NM, Komor AC, Rees HA, Packer MS, Badran AH, Bryson DI, et al. Programmable base editing of $A * T$ to $G^{*} C$ in genomic DNA without DNA cleavage. Nature. 2017;551:464-71.

21. Hu JH, Miller SM, Geurts MH, Tang W, Chen L, Sun N, et al. Evolved Cas9 variants with broad PAM compatibility and high DNA specificity. Nature. 2018;556:57-63.

22. Nishimasu H, Shi X, Ishiguro S, Gao L, Hirano S, Okazaki S, et al. Engineered CRISPR-Cas9 nuclease with expanded targeting space. Science. 2018;361:1259-62.

23. Sayers EW, Cavanaugh M, Clark K, Ostell J, Pruitt KD, KarschMizrachi I. GenBank. Nucleic Acids Res. 2019;47:D94-D99.

24. Joung J, Konermann S, Gootenberg JS, Abudayyeh OO, Platt RJ, Brigham MD, et al. Genome-scale CRISPR-Cas9 knockout and transcriptional activation screening. Nat Protoc. 2017;12:828-63.

25. Kweon J, Kim DE, Jang AH, Kim Y. CRISPR/Cas-based customization of pooled CRISPR libraries. PLoS ONE. 2018;13:e0199473.

26. Kim Y, Kweon J, Kim A, Chon JK, Yoo JY, Kim HJ, et al. A library of TAL effector nucleases spanning the human genome. Nat Biotechnol. 2013;31:251-8. 